\title{
La participación como enfoque integral de aprendizaje para una práctica crítica en la gestión de proyectos y procesos de cooperación al desarrollo. El caso de un Máster en la Universitat Politècnica de València.
}

\section{Belda-Miquel, Sergio ${ }^{a}$, Peris Blanes, Jordi ${ }^{\mathrm{b}}$ y Cuesta Fernández, Iván}

${ }^{a}$ INGENIO. Ciutat Politècnica de la Innovació - Edifici 8E $5^{\text {a }}$ Planta, Universitat Politècnica de València - serbelmi@ingenio.upv.es ${ }^{b} I N G E N I O . j p e r i s b @$ dpi.upv.es ${ }^{c}$ University of Edimbourgh. S1259893@sms.ed.ac.uk

\begin{abstract}
There is a growing concern about the role of the university in the education of international development professionals. For a number of actors, it should play a central role in order to move current managerial trends in the international development sector towards a more transformative and reflective practice of international development projects management. In the paper, we depart from the idea that postgraduate courses can promote the emergence of more critical and reflective professionals, if they assume participation as a key issue in the curricula and in the methodological approach.

We will deep into a particular case study, a Master in development management in the Universitat Politècnica de València. We will see how participation is considered at different levels, as a key pedagogical strategy, for the emergence competences for a reflective and transformative practice of international cooperation. We will also see the outputs of this Master in terms of the learning experienced by students in this regards, and we will address the limitations and opportunities that the Master faces in its strategy. Results are built on interviews to students, teachers and other relevant stakeholders, as well as in the results of external and internal evaluations and reports on the Master's performance.
\end{abstract}

Keywords: development management; international cooperation; participation; learning; Master programme; critical practice

\footnotetext{
Resumen

Existe un creciente interés en los debates sobre el rol que la Universidad debe jugar en la formación de profesionales de la cooperación internacional al desarrollo. Desde cierta perspectiva crítica, se ha expuesto que debería jugar un rol fundamental para alejar la gestión de proyectos y procesos de desarrollo del actual enfoque gerencial, para abrazar en cambio una perspectiva más crítica, reflexiva y sensible a la complejidad de los procesos de cambio social. En este trabajo, asumimos esta posición. También
} 
consideramos que la participación como enfoque de enseñanza aprendizaje y como forma de construcción del currículum, puede ser muy relevante para una formación que contribuya a la formación de estos profesionales críticos y reflexivos.

Nos aproximaremos a un caso de estudio particular, el de los estudios de postgrado en gestión de proyectos y procesos de desarrollo impartidos en el Universitat Politècnica de València desde 2007. El caso ilustra como la participación, considerada desde una perspectiva integral, puede contribuir a la emergencia de competencias centrales para esta práctica crítica de la cooperación. Sobre la base de entrevistas y talleres con alumnado, profesorado y otros actores del sistema de cooperación, así como de información secundaria, analizaremos los resultados de esta estrategia, asi como las limitaciones y oportunidades que ha enfrentado.

Palabras clave: gestión del desarrollo; cooperación internacional; participación; aprendizaje; Máster; práctica crítica

\section{Introducción. Aprendizaje del pensamiento crítico para el pensamiento crítico en la gestión del desarrollo}

En el ámbito de la gestión del desarrollo, existe un intenso debate sobre el rol de la formación superior en la educación de profesionales del desarrollo, que conecta con reflexiones más profundas acerca de qué capacidades son necesarias para la gestión del desarrollo, y cómo pueden desarrollarse en un proceso de educación formal (Clarke y Oswald, 2010).

Existe en esta discusión una corriente crítica, que comprende autores que opinan que la tendencia mayoritaria en la formación en gestión del desarrollo internacional está dominada por una perspectiva puramente instrumental y poco crítica. Esta perspectiva se sostendría en una visión de la educación puramente racionalista, que entiende que los procesos educativos son procesos lineales de adquisición y aplicación de conocimiento. Esta visión se sustentaría además en una fe ciega en las bondades de la modernización y en la posibilidad de controlar el cambio social mediante los instrumentos adecuados (Johnson y Thomas, 2007). Como Peris et al. (2013) mencionan, esta perspectiva hace que los procesos educativos se centren en proveer a los estudiantes de instrumentos tecnocráticos de gestión, de modo que tengan las herramientas para alcanzar de manera eficiente objetivos prefijados de desarrollo mediante una secuencia lineal de pasos, o al menos mediante una planificación estricta (Clarke and Oswald, 2010). Para los críticos, esta perspectiva, que se puede llamar gerencial, ve problemas complejos y profundamente políticos como son los del desarrollo como problemas puramente técnicos y de gestion. Ve a su vez a los profesionales del desarrollo como simples técnicos que deben ofrecer soluciones racionales, en lugar de cómo personas que van a actuar en contextos siempre complejos y cambiantes,

(cc) EY-NC-ND 2016, Universitat Politècnica de València 
embebidos en relaciones de poder con frecuencia poco visibles. Para estos críticos, estas perspectivas tecnocráticas y gerenciales no contribuirían a abordar las causas estructurales de la pobreza y la exclusión sino que, al contrario, reforzarían relaciones desiguales de poder.

Por todo lo anterior, se puede sostener que resulta necesario repensar la formación de los profesionales del desarrollo, a fin de contribuir a generar perfiles con una perspectiva más crítica y compleja. En este trabajo se construye por ende sobre una perspectiva alternativa de la gestión del desarrollo y de los procesos educativos necesarios para generar capacidades adecuadas para esta práctica crítica de la gestión. Esta perspectiva considera que los gestores del desarrollo deberían poner en el centro la naturaleza siempre compleja y política de los procesos de desarrollo, en los que las relaciones de poder juegan un lugar fundamental, procesos que son permanentemente modelados y re-modelados por intereses e influencias cambiantes (McCourt, 2008; Eyben et al, 2015). Como Belda et al. (2012) señalan, esta perspectiva nos hace pensar en otro tipo de capacidades necesarias para las personas que gestionan el desarrollo: Éstas deberían ante todo ser capaces de situarse en procesos siempre cambiantes y ser capaces de desvelar y transformar las relaciones desiguales de poder -que pueden ser más o menos visibles- (Townley, 2001; Mosse, 2005), para orientar consciente y críticamente su acción hacia el bien común (Walker and McLean, 2013; Boni-Aristizábal and Calabuig-Tormo, 2015).

Esta perspectiva implicaría que los programas de educación superior en gestión del desarrollo, en lugar de centrarse en el desarrollo de habilidades para el manejo de instrumentos gerenciales de gestión, deberían focalizarse en generar competencias para entender, desvelar y moverse en redes complejas de relaciones de poder; en desarrollar la capacidad de realizar análisis políticos propios de los contextos; en generar competencias para navegar la complejidad y el cambio permanente; en generar la capacidad en el alumnado para adaptarse y aprender en la constante adaptación del cambio, para reflexionar permanentemente sobre lo que se hace y sobre por qué se hace (Pettit, 2010; Mowles et al., 2008; Clarke and Oswald, 2010). Serían estas las competencias para lo que puede ser llamada una práctica crítica del desarrollo (Clarke y Oswald, 2010).

Distintos autores han trabajado sobre la base de una perspectiva parecida, y han afirmado que existen distintos métodos y aproximaciones pedagógicas para desarrollar este tipo de capacidades (ver ejemplos específicos en Fisher, 2010; Jackson, 2010; Ortiz Aragon, 2010, Pearson, 2010; Pettit, 2010; Soal, 2010), que van desde el aprendizaje experiencial a métodos creativos que apelan a las emociones y el uso del cuerpo. Entre estos, varios autores ponen en el centro la importancia de los métodos y enfoques participativos de apredizaje (Pettit, 2010). En este sentido, consideran la participación no sólo como un instrumento de aprendizaje y no sólo como una capacidad, sino como todo un proceso de empoderamiento y transformación personal y colectiva, necesario no sólo para un aprendizaje crítico sino, más en general, para la construcción de ciudadanos y sistemas más democráticos (Gaventa, 2006).

(cc) EY-NC-ND 2016, Universitat Politècnica de València

Congreso In-Red (2016) 


\section{Objetivos}

En este trabajo nos referimos al caso particular de los estudios de postgrado en gestión de proyectos y procesos de cooperación al desarrollo en la Universitat Politècnica de València (UPV). Esta experiencia comprende el Máster en Políticas y Procesos de Desarrollo, Especialidad en Gestión de Proyectos y Procesos de Desarrollo, que se ha impartido como Máster Título Propio en la UPV desde 2007, y que fue el germen del título oficial que lo reemplazó en 2011, ya adaptad a los requisitos de Bolonia, el Máster Universitario en Cooperación al Desarrollo, Especialidad en Gestión de Proyectos y Procesos de Desarrollo, todavía activo. Podemos considerar que ambos títulos responden a un mismo proyecto pedagógico, al que nos referiremos como Máster en gestión del desarrollo. En este trabajo veremos cómo la participación ha jugado un rol central, en múltiples aspectos y niveles, en este Máster, para la formación de capacidades relevantes para el ejercicio de una práctica crítica de gestión de proyectos y procesos de cooperación y desarrollo internacional. En este sentido, entendemos que se trata de una experiencia innovadora y potencialmente inspiradora, tanto por sus resultados positivos como por sus limitaciones.

\section{Desarrollo de la innovación}

\subsection{La experiencia en Máster en gestión del desarrollo en la UPV}

El Máster en Políticas y Procesos de Desarrollo tenía una carga docente equivalente a 66 créditos ECTS. Por su parte el Máster Universitario en Cooperación al Desarrollo tiene una carga algo mayor, de 90 ECTS. Ambos se centran en la misma especialización en gestión de proyectos y procesos de desarrollo. En las dos fases del Máster se ha combinado un primer año de aprendizaje en la UPV con un segundo año en el que el alumnado realiza prácticas externas y elabora el Trabajo Final de Máster.

El Máster nace y se desarrolla en una universidad de orientación técnica, donde todavía es muy frecuente una docencia muy centrada en la clase magistral, donde se da una visión muy instrumental de las capacidades que la Universidad debe desarrollar en sus alumnos, así como una visión de la función de la Universidad muy centrada en la empleabilidad de las personas egresadas. Por otro lado, el perfil del personal docente del Máster es muy variado, con varias personas con un perfil en ingeniería, pero también en varias ramas de las ciencias sociales y las humanidades. La estructura y enfoque pedagógico del proyecto del Máster ha tenido continuidad en el tiempo, si bien ha experimentado cambios, en buena parte dado la constante retroalimentación recibida en distintos espacios de seguimiento, evaluación, reflexión y discusión, en los cuales docentes, alumnados, miembros de ONG y otros actores han tomado parte. Estos espacios han sido de vital importancia para ir permanentemente innovando y transformando el proyecto pedagógico.

(cc) EY-NC-ND 2016, Universitat Politècnica de València 


\subsection{Metodología de la investigación}

La estrategia de investigación fue puramente cualitativa. Se basa en el estudio de caso, con una orientación comprensiva, desde un paradigma interpretativo-constructivista, siguiendo la clasificacióin de Lincoln et al. (2010).

En relación a los datos empleados, para el trabajo se analizó tanto información primaria como secundaria. En cuento a la primaria, se contó con 14 entrevistas realizadas a alumnos que habían concluido el Máster (o estaban sólo a falta del TFM). En las entrevistas se preguntaba, por un lado, por las competencias que ellos creían haber desarrollado en distintos aspectos relacionados con la definición de práctica crítica del de desarrollo que hemos formulado: manejo de la complejidad; análisis del poder; capacidad de adaptación, aprendizaje y reflexión permanentes. Se pedía también que mencionaran espacios, momentos y métodos del Máster que percibieran como que han contribuido a desarrollar la competencia, así como que se narraran situaciones de su práctica profesional o vida personal que permitieran reconocer que la competencia se había efectivamente desarrollado.

La información seundariaanalizada incluyó 3 informes de evaluación realizados por profesionales externos al Máster (Cascant, 2010; Frediani y Terol, 2011; Guijarro, 2012). En ellos, se evaluaba tanto el proceso, resultados y métodos de aprendizaje, como las relaciones con otros actores que tiene el Máster. Estos informes se construyeron a partir de más de 20 entrevistas semi-estructuradas a estudiantes, profesorado, personas miembros de ONG y otros actores del sector de la cooperación. También se realizaron talleres en los que participaron estos grupos. Además, se analizaron algunos trabajos desarrollados por el alumnado. Finalmente, se emplearon resultados de anteriores investigaciones de los autores sobre el tema, disponibles en Belda et al (2012), Peris et al. (2013).

Se realizó un análisis de contenido tanto de la información primaria como secundaria, con un sistema de categorías en base a los distintos espacios de participación en relación al Máster (diseño de currículum, metodología, competencia adquirida, contenido, método de evaluación, ver sección 3.3). Se trataron de encontrar tendencias generales sobre el rol de la participación en estos ámbitos, en relación a la formación de profesionales para una práctica crítica del desarrollo. Se identificaron tanto los resultados de la incorporación de la participación en distintos ámbitos como las oportunidades y limitaciones generales que afronta. Esta lógica de análisis queda reflejada en la presentación de los resultados.

\subsection{La participación en el Máster: Estrategias, herramientas y espacios.}

En la experiencia en máster en gestión del desarrollo en la UPV, la participación se ha introducido como estrategia fundamental de distintos modos:

- En primer lugar, la participación ha jugado un rol fundamental en el diseño de los currículums (tanto del Máster título propio como del oficial). A través de diferentes talleres y discusiones on-line, organizaciones y profesionales del sector de la cooperación pudieron dar su visión acerca de qué conocimientos, 
habilidades, valores, actitudes, etc. esperarían que se desarrollaran en las personas que cursaran el Máster. Además, los borradores de programas del Máster fueron sometidos a evaluación por pares de profesorado y personal investigador de varias universidades europeas y latinoamericanas. Todos estos inputs jugaron un rol fundamental en la definición final de los currículums docentes. Si bien el diseño general del Máster no puede ser modificado una vez validado, las evaluaciones anuales sí han permitido ir incorporando cambios en contenidos y métodos (ver al final de la sección).

- En segundo lugar, la participación ha sido esencial en la definición del enfoque pedagógico general y de la metodología de enseñanza. A modo de ejemplo, un número elevado de distintas técnicas participativas se emplean tanto en clase o para el trabajo fuera de ella, que promueven la discusión, deliberación y reflexión: del uso real de herramientas de planificación participativa y el diseño y desarrollo de proyectos de investigación-acción participativa al uso de herramientas de teatro y dramatización como disparadores de la participación. Las dinámicas de grupo son en general muy frecuentes en clase, teniendo presencia en todas las asignaturas y en casi todas las sesiones presenciales. La participación activa en clase, y el facilitar la participación de los demás, es objeto de evaluación en todas las asignaturas, con un peso de al menos el $10 \%$ de la nota final. Para ello, se emplea tanto la evaluación por parte del profesor (generalmente empleando rúbricas propias), la evaluación entre alumnos, y la autoevaluación. Con frecuencia se triangula la información de estas tres fuentes.

- En tercer lugar, la participación es a su vez una competencia clave a ser desarrollada por el alumnado, definida en varios sentidos complementarios: la participación como rasgo propio de una gestión responsable, reflexiva y eficiente de proyectos y procesos de desarrollo; la participación como rasgo propio de la ciudadanía activa y comprometida (que se espera desarrollar en el o la estudiante); la participación como capacidad de generar participación. Esta competencia está presente en la mayoría de asignaturas del Máster.

- Cuarto, la participación tiene un rol importante como contenido en distintas asignaturas. Es una cuestión presente en buena parte de las materias: por ejemplo, se trabaja la historia de la participación en el desarrollo, la participación como enfoque de planificación, la participación como técnica y enfoque de investigación, etc.

- En quinto lugar, la participación juega un rol fundamental en la evaluación continua del propio Máster. Se han generado para ello varios espacios y mecanismos. Por ejemplo, los alumnos realizan evaluaciones participativas en clase con frecuencia, sobre las distintas asignaturas y unidades temáticas; un comité de seguimiento con representación de alumnado y profesorado se reúne periódicamente; y un consejo asesor compuesto por varias ONG y otros actores clave del sector valenciano de la cooperación ha estado reuniéndose al menos una

(c)) EY-NC-ND 2016, Universitat Politècnica de València 
vez al año para dar orientaciones al Máster, sobre la base de la actualidad del sector.

Cabe decir que estas evaluaciones han dado lugar a acciones de mejora frecuentes por parte del Máster. Por ejemplo, y sobre la base de las percepciones y demandas recogidas en las evaluaciones participativas, se ha transversalizado el enfoque de género en varias aisgnaturas, se han incorporado seminarios sobre temas específicos (como trabajo en equipo), se abrió un proceso de reflexión sobre el rol del consejo asesor, o se dio más peso a la investigación acción participativa en los contenidos.

\subsection{Limitaciones y oportunidades}

En cualquier caso, la introducción de la participación como cuestión clave, como proceso de diseño curricular, enfoque pedagógico, contenido o herramienta de evaluación de la docencia para el aprendizaje y la innovación, no es una cuestión sencilla, dadas limitaciones tales como la cultura académica o la naturaleza del sector de la cooperación en el entorno de Valencia y España (Peris et al., 2013), como veremos a continuación.

En primer lugar, es conocido que la cultura académica en España todavía promueve en buena medida cierta actitud pasiva hacia el aprendizaje por parte del alumnado. Esto significa que buena parte de los alumnos deben familiarizarse con un enfoque y tipo de técnicas a los que no están siempre acostumbrados. En ocasiones puntuales, pueden en un principio hasta ser vistos como poco útiles. En cualquier caso, buena parte de los estudiantes, sobre todo aquellos que tienen experiencia en el mundo del activismo o de las ONG, que suelen ser numerosos, suelen presentar la actitud contraria, se muestran entusiastas ante estos enfoques, y contribuyen a generar dinámicas de aprendizaje muy positivas con métodos participativos.

En segundo lugar, la cultura de la docencia universitaria en centros politécnicos en España tampoco ha tenido de forma mayoritaria un enfoque participativo. Esto también ha introducido por momentos algunas limitaciones, tales como la dificultad de contar con espacios físicos adecuados para el desarrollo de talleres participativos. Sin embargo, cabe decir que la actual estrategia de muchos centros, como la UPV, es la de incentivar este tipo de enfoques, algo que ha generado también legitimidad, reconocimiento y facilidades al Máster.

En tercer lugar, en el propio sector de la cooperación en España existen muchas organizaciones y agencias con una perspectiva y funcionamiento muy gerenciales y burocratizados, con buena parte del personal dedicado casi exclusivamente a la gestión técnica y financiera de proyectos, sin mayor reflexión ni capacidad de influencia. Este hecho crea en el alumnado la sensación de que lo que se aprende en el Máster (por ejemplo, todas las cuestiones relacionadas con las relaciones de poder, la complejidad y la reflexión) están muy alejadas de día a día de las organizaciones. Además, algunas organizaciones pueden desarrollar la idea de que el Máster no está desarrollando las competencias en el uso de herramientas de gestión técnica y financiera que el personal técnico del sector necesita. En cambio, el Máster mantiene una relación muy estrecha y productiva con un cierto

2016, Universitat Politècnica de València

Congreso In-Red (2016) 
número de organizaciones que tienen perspectivas afines y más críticas sobre el desarrollo, lo que genera espacios de encuentro y confianza muy relevantes.

\section{Resultados: Impactos del uso de la participación para el aprendizaje crítico.}

A pesar de las limitaciones y problemas señalados, la mayor parte del alumnado valora enormemente la importancia que da el Máster a la participación, así como su relevancia para el desarrollo de competencias.

La participación es valorada, por un lado, como un resultado del proceso de aprendizaje - el aprender a participar y promover la participación; por otro, como facilitador del aprendizaje (Belda et al., 2012): Por una parte, el alumnado parece reconocer que ha desarrollado capacidades para participar, para gestionar de manera participativa y para promover la participación, y que este hecho ha podido experimentarlo en la fase de prácticas del Máster o en su vida como activista o profesional de la cooperación. Por otra parte, el alumnado parece en buena parte identificar que los métodos participativos han sido centrales para desarrollar algunos conocimiento, habilidades, actitudes, etc. clave para lo que puede considerarse una gestión crítica del desarrollo, y que de otro modo difícilmente podrían haber sido trabajadas. Por ejemplo, se menciona que sin las dinámicas participativas de grupo, o sin realizar investigación-acción participativa, habría sido mucho más difícil trabajar la capacidad de identificar relaciones de poder y formas de transformarlas. Esto no significa que no haya diversidad entre el alumando en la intensidad y procesos de aprendizaje, viéndose desigualdades entre personas, no siempre fáciles de abordar por el profesorado.

Sobre resultados y relaciones de poder, es interesante que el alumnado menciona que las dinámicas de poder dentro de la clase habían sido clave para modelar los procesos y resultados del aprendizaje al emplear métodos participativos. Por ejemplo, con frecuencia se menciona que la diversidad de perfiles y niveles de experiencia entre el alumnado del Máster es un elementos importante para impulsar el aprendizaje a través de la participación. Sin embargo, según algunas personas entrevistadas, las diferencias entre los conocimientos y experiencia previa entre unos y otros alumnos puede con frecuencia generar dinámicas de poder que inhiben la participación y limitan el aprendizaje compartido.

Por otro lado, los resultados demuestran que la participación resulta de gran importancia en el proceso de aprendizaje crítico de los alumnos, en espacios con frecuencia no considerados por el profesorado. Por ejemplo, la participación en organizaciones sociales mientras se está cursando el Máster parece ser de mucha importancia para que se den aprendizajes relevantes. Sin embargo, estos espacios, así como la experiencia previa del alumnado, no son siempre han sido aprovechados en el Máster. Por otro lado, los requisitos de la adaptación a Bolonia han limitado también la posibilidad por parte del alumnado de participar en organizaciones. Esta situación, paradójicamente, ha limitado el potencial de esta forma de aprendizaje.

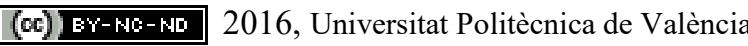


Finalmente, cabe también decir que resulta complicado atribuir a un cierto enfoque o méodos docentes los resultados del aprendizaje. Sin embargo, dadas las percepciones de los alumnos, dado que la participación es un enfoque clave del Máster, y dado que parecen desarrollarse en el alumnado competencias relevantes para una gestión crítica del desarrollo mencionadas (analizar y transformar relaciones de poder, reflexividad, capacidad de adaptación, moverse en contextos complejos, etc.), parece plausible afirmar que la participación en sus múltiples dimensiones puede tener un rol fundamental en procesos de aprendizaje para generar profesionales críticos en la práctica del desarrollo.

\section{Conclusiones}

La experiencia señalada ofrece algunas interesantes lecciones y reflexiones que pueden ser relevantes para otros máster en gestión del desarrollo así como, más en general, para otras máster en gestión de proyectos o en otras disciplinas. Entre estas reflexiones, podemos mencionar, las siguientes:

- La inclusión efectiva, genuina y útil de la participación en un Máster orientada a la generar profesionales y ciudadanía con capacidad critica, parece pasar por considerar la participación en múltiples niveles y aspectos: como proceso de diseño y evaluación, como contenido, como competencia, como enfoque y como técnicas de aprendizaje. Parece que esto es relevante en la experiencia del alumnado del Máster analizado. Además, parece que la participación está modelada por una multiplicidad de factores, tales como los vistos de la diversidad en el perfil de los estudiantes y las relaciones entre ellos, o la multiplicidad de espacios de participación durante la duración del Máster (tales como en las organizaciones a las que pertenecen), que deben ser en lo posible considerados, y aprovechados para fortalecer procesos de aprendizaje (por ejemplo, conectando el aprendizaje planificado en el Máster y el no planificado en las organizaciones) y evitar las limitaciones (por ejemplo, de las relaciones desiguales de poder entre alumnos).

- La cultura del alumnado, profesorado, de la universidad y del sector del desarrollo en relación a la participación puede ser una limitación importante para le incorporación de la misma como elemento clave en el aprendizaje. Sin embargo, la tendencia en la universidad y en el sector educativo en general, así como en otros sectores o en la propia elaboración de la política pública, esta resituando la importancia de la participación de forma acelerada, de modo que nuevas oportunidades estarían emergiendo para incorporar la participación con eje central de los proyectos educativos.

- Discutir sobre la participación en un master en gestión del desarrollo nos remite, como ha sido el inicio de este artículo, a la cuestión sobre qué es importante en la gestión de proyectos y procesos de desarrollo, y sobre qué es y cómo construir una perspectiva y práctica críticas de la gestión. En un sentido más amplio, el debate sobre la participación en la educación superior nos debería ayudar a reflexionar

(cc) EY-NC-ND 2016, Universitat Politècnica de València

Congreso In-Red (2016) 
críticamente sobre el propio sentido de la educación y de la práctica profesional en distintos ámbitos, en una sociedad democrática.

\section{Referencias}

BELDA, S., BONI, A., PERIS, J. y TEROL, L. (2012). “Capacity development for emancipator social change. Reimaging university learning and teaching for critical development practitioners" en Journal of International Development, vol. 24, p. 571-584.

BONI-ARISTIZABAL, A. y CALABUIG-TORMO, C. (2016). "Enhancing pro-public-good professionalism in technical studies" en Higher Education (en publicación)

BONI-ARISTIZABAL, A., CALABUIG-TORMO, C. y PELLICER SIFRES, V. (2015). Transforming the system from within: the experience of the Development Cooperation. Informe sin publicar.

CLARKE, P., y OSWALD, K. (2010). "Why reflect collectively on capacities for change?" en IDS Bulletin, issue 41, p. 1-12.

CASCANT, M.J. (2010). Evaluación de los tres primeros años del Máster en Políticas y Procesos de Desarrollo. Informe para el Máster en Políticas y Procesos de Desarrollo, Universitat Politècnica de València, València, Julio 2010

EYBEN, R., GUIJT, I., ROCHE, C. y C. SHUTT (Eds.) (2015). The Politics of Evidence and Results in International Development: Playing the game to change the rules? Rugby: Practical Action Publishing.

EYBEN, R. (2005). "Donors' learning difficulties: Results, relationships and responsibilities" en IDS Bulletin, vol. 36, p. 98-107.

FREDIANI, A. y TEROL, L. (2011). Informe Evaluación $4^{\text {a }}$ Edición del Máster en Políticas y Procesos de Desarrollo. Informe para el Máster en Políticas y Procesos de Desarrollo, Universitat Politècnica de València, València, Julio 2011.

GUIJARRO, D (2012). Informe de Evaluación del Máster en Políticas y Procesos de Desarrollo 2010/2011. Informe para el Máster en Políticas y Procesos de Desarrollo, Universitat Politècnica de València, València, Julio 2012.

GULRAJANI, N. (2010). "New vistas for development management: examining radical-reformist possibilities and potential" en Public Administration and Development, vol. 30, p. 136-148.

JOHNSON, H. y THOMAS, A. (2007). "Individual learning and building organizational capacity for development" en Public Administration and Development, vol. 27, p. 39-48.

LINCOLN Y., LYNHAM S. A. y GUBA E. G. (2011). «Paradigmatic controversies, contradictions, and emerging confluences, revisited ». En Denzin N. K. y Lincoln Y. S. (Eds.), The SAGE handbook of qualitative research. LA: SAGE.

MCCOURT, W., y GULRAJANI, N. (2010). "The future of development management: Introduction to the Special Issue" en Public Administration and Development, vol.30, p. 81-90.

MCFARLANE, C. (2006). "Crossing borders: development, learning, and the North-South divide" en Third World Quarterly, vol. 27, p. 1413-1437.

MOSSE, D. (2005). Cultivating development: An ethnography of aid policy and practice. London: Pluto Press.

(cc) EY-NC-ND 2016, Universitat Politècnica de València 
ORTIZ ARAGON, A. (2010). "A case for surfacing theories of change for purposeful organizational capacity development” en IDS Bulletin, vol.41, p. 36-46.

PERIS, J., BELDA, S., y CUESTA, I. (2013). "Educating development professionals for reflective and transformative agency”. En Boin A \& Walker M. (Eds.). Human Development and Capabilities: Re-imagining the University of the Twenty-first Century, London and New York: Routledge.

SOAL, S. (2010). "The more things change, the more they stay the same?", en IDS Bulletin, vol.41, p. $128-137$.

PEARSON, J. (2010). "Pushing at a half-open door" en IDS Bulletin, vol. 41, p. 118-127.

PETTIT, J. (2006). "Power and pedagogy: Learning for reflective development practice" en IDS Bulletin, vol.37, p. 69-78.

TOWNLEY, B. (2001). "The cult of modernity" en Financial Accountability and Management, vol.17, p. 303-310.

WALKER, M. y MCLEAN, M. (2013). Professional Education, Capabilities and the Public Good. London: Routledge. 